$01,13,19$

\title{
Сила трения и радиационный теплообмен в системе двух параллельных пластин при их относительном движении: следствия теории Левина-Полевого-Рытова
}

\author{
(C) Г.В. Дедков, А.А. Кясов \\ Кабардино-Балкарский государственный университет, \\ Нальчик, Россия \\ E-mail: gv_dedkov@mail.ru
}

(Поступила в Редакцию 27 февраля 2018 г.

В окончательной редакции 23 мая 2018 г.)

Показано, что фундаментальные результаты, полученные в работах Левина, Полевого, Рытова (1980) и Полевого (1990), базирующиеся на теории электромагнитных флуктуаций Левина и Рытова, адекватно описывают скорость радиационного теплообмена и силу трения в системе двух толстых параллельных пластин, находящихся в относительном движении, в полном согласии с более поздними результатами других авторов. Численные оценки величины силы трения для хороших металлов в приближении Друде оказываются в $10^{7}$ раз выше первоначальной оценки Полевого, причем сила трения возрастает с увеличением проводимости пластин (увеличением времени релаксации электронов при понижении температуры).

DOI: 10.21883/FTT.2018.12.46718.043

\section{1. Введение}

Флуктуационно-электромагнитная теория Левина и Рытова [1] является развитием теории Рытова [2]. В [1], спектр электромагнитных флуктуаций нагретого тела на произвольном расстоянии от него выражается через смешанные потери двух точечных дипольных источников, находящихся вблизи тела, которые находятся в результате решения регулярной электродинамической задачи. Это является сущностью обобщенного закона Кирхгофа, представляющего форму флуктуационнодиссипационной теоремы.

В рамках теории [1] были получены выражения для скорости радиационного теплообмена между двумя полубесконечными средами (толстыми пластинами), разделенными плоской вакуумной щелью конечной ширины $[3,4]$, а также диссипативной силы трения, возникающей при латеральном относительном движении одной из пластин [5]. Первый расчет радиационного теплообмена между покоящимися пластинами (в рамках теории [2]) был выполнен Полдером и ван Ховом [6] в более простом случае двух одинаковых пластин и малой разности температур между ними. В отличие от этого, в $[3,4]$ предполагалось, что материалы пластин 1 и 2 характеризуются однородными и изотропными свойствами с диэлектрическим проницаемостями и магнитными восприимчивостями $\varepsilon_{1}, \mu_{1}$ и $\varepsilon_{2}, \mu_{2}$, являющимися комплексными функциями частоты $\omega$. Кроме того, рассматривался также случай анизотропных сред. Позднее, задача радиационного теплообмена рассматривалась многими авторами, но формула для потока тепловой энергии воспроизводилась, как правило, либо без ссылок на $[3,4]$ (см., например, [7, 8]), либо в другой эквивалентной форме $[9,10]$.
Ситуация с работой [5] сложилась значительно более драматически: в конечных формулах для силы трения в линейном приближении по скорости появилась зависимость $F \propto V / c^{3}$ ( $c$ - скорость света в вакууме), тогда как позже несколько авторов получали линейные по скорости $V$ и не зависящие от $c$ выражения для этой силы (при конечной температуре пластин) [11,12], или зависимость $F \sim V^{3}$ в квантовом пределе нулевой температуры [13]. Эти противоречия „подлили масла в огонь“ длительной дискуссии относительно величины диссипативной силы, начавшейся еще ранее [14], и не завершенной до сих пор $[10-13,15-20]$ (см. ссылки в $[19,20])$.

В этой работе мы покажем, что базисные результаты для силы трения и скорости радиационного теплообмена, полученные в [3-5], полностью согласуются со всеми результатами других авторов, полученными позже, тогда как зависимость $F \propto V / c^{3}$ и очень малая численная величина фрикционного напряжения $10^{-14} \mathrm{~N} / \mathrm{m}^{2}$, полученная для металлических пластин при комнатной температуре, ширине щели $10 \mathrm{~nm}$ и относительной скорости $1 \mathrm{~m} / \mathrm{s}$, обусловлена использованным приближением для материальных свойств взаимодействующих тел. Мы провели независимые численные расчеты сил трения для пластин из золота, используя диэлектрическую проницаемость Друде, как в низкочастотном приближении $\varepsilon(\omega)=i 4 \pi \sigma / \omega(\sigma-$ фиксированная проводимость, аналогично [5]), так и в общем случае $\varepsilon(\omega)=1-\frac{\omega_{p}^{2}}{\omega(\omega+i / \tau)}$ $\left(\omega_{p}\right.$ и $\tau-$ плазменная частота и время релаксации электронов). В обоих случаях полученные значения сил трения выше в $10^{7}$ раз, чем в [5] при тех же условиях. Кроме того, оказывается, что сила трения металлических пластин значительно возрастает при температурах 


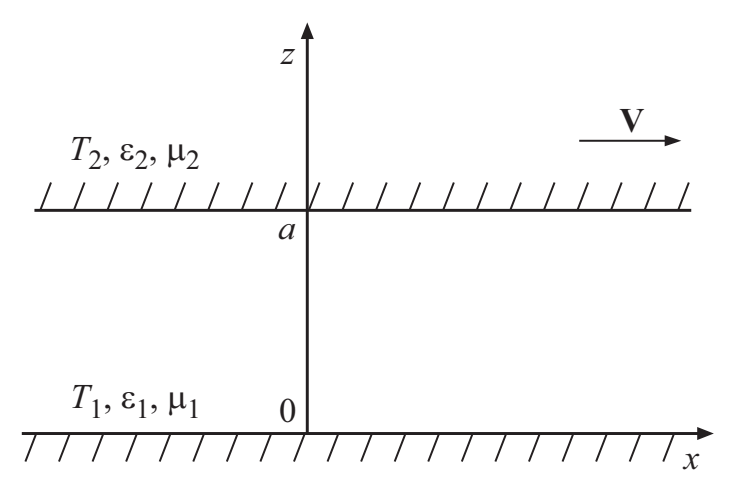

Рис. 1. Конфигурация системы.

$T<50 \mathrm{~K}$, если зависимость времени релаксации электронов от $T$ определяется законом Блоха-Грюнайзена.

\section{2. Постановка задачи и общее выражение для тангенциальной силы, полученное Полевым}

В конфигурации системы, использовавшейся в [5], декартова лабораторная система координат, связанная с пластиной 1 (рис. 1) выбрана так, что ось $z=x_{3}$ ортогональна границам пластин, ось $x=x_{1}$, без потери общности, параллельна скорости $\mathbf{V}$ пластины 2, а ось $y=x_{2}$ (не показанная на рис. 1) ортогональна осям $x$ и $z$. Температуры пластин считаются постоянными и равными $T_{1}$ и $T_{2}$ соответственно.

Следуя [5], результирующие плотности сил $\mathbf{F}_{1}$ и $\mathbf{F}_{2}$, действующих на единицу площади поверхности пластин 1 и 2, отличаются только знаком: $\mathbf{F}_{1}=-\mathbf{F}_{2}=\mathbf{V} F / V$, где $F-$ модуль диссипативной тангенциальной силы, действующей на единицу площади контакта движущейся пластины 2 в лабораторной системе отсчета, связанной с покоящейся пластиной 1. Сила $F$ выражается в терминах потоков тепла $P_{1}$ и $P_{2}$ от пластин 1 и 2 (на единицу площади), где $P_{1}$ находится с помощью вектора Пойнтинга в системе отсчета пластины 1 , а тепловой поток $P_{2}$ от пластины 2 находится в ее собственной системе покоя

$$
F=\frac{1}{V}\left(P_{1}+P_{2} / \gamma\right)
$$

где $\gamma=1 /\left(1-u^{2}\right)^{1 / 2}, u=V / c$. Тепловые потоки $P_{1}$ и $P_{2}$ определяются выражениями

$$
\begin{aligned}
& P_{1}=\frac{\hbar}{8 \pi^{3}} \int_{-\infty}^{\infty} d \omega \int d^{2} k\left(\frac{\omega}{|\omega|}-\frac{\tilde{\omega}}{|\tilde{\omega}|}\right) \omega M(\omega, \mathbf{k}, \mathbf{u}) \\
& +\frac{1}{4 \pi^{3}} \int_{-\infty}^{\infty} d \omega \int d^{2} k\left[\frac{\Pi\left(T_{1}, \omega\right)}{\omega}-\frac{\Pi\left(T_{2}, \tilde{\omega}\right)}{\tilde{\omega}}\right] \omega M(\omega, \mathbf{k}, \mathbf{u}),
\end{aligned}
$$

$$
\begin{aligned}
& P_{2}=-\frac{\hbar}{8 \pi^{3}} \int_{-\infty}^{\infty} d \omega \int d^{2} k\left(\frac{\omega}{|\omega|}-\frac{\tilde{\omega}}{|\tilde{\omega}|}\right) \tilde{\omega} M(\omega, \mathbf{k}, \mathbf{u}) \\
& -\frac{1}{4 \pi^{3}} \int_{-\infty}^{\infty} d \omega \int d^{2} k\left[\frac{\Pi\left(T_{1}, \omega\right)}{\omega}-\frac{\Pi\left(T_{2}, \tilde{\omega}\right)}{\tilde{\omega}}\right] \tilde{\omega} M(\omega, \mathbf{k}, \mathbf{u}),
\end{aligned}
$$

где $\hbar-$ постоянная Планка, $\mathbf{k}=\left(k_{1}, k_{2}\right)$ - двумерный волновой вектор, параллельный границам щели, $\quad \tilde{\omega}=\gamma(\omega-\mathbf{k V}), \quad|\mathbf{V}|=V, \quad|\mathbf{u}|=u, \quad \Pi(T, \omega)=$ $=\hbar|\omega| /\left(\exp \left(|\omega| / \omega_{T}\right)-1\right), \omega_{T}=T / \hbar, T-$ температура в энергетических единицах, а интегрирование выполняется по всей области изменения волновых векторов. Функция $M(\omega, \mathbf{k}, \mathbf{u})$ имеет вид

$$
\begin{aligned}
M= & \frac{4|q|^{2}}{|Q|^{2}}\left[\operatorname{Im}\left(\frac{q_{1}}{\varepsilon_{1}}\right) \operatorname{Im}\left(\frac{\tilde{q}_{2}}{\tilde{\varepsilon}_{2}}\right)(1+\beta)\left|Q_{\mu}\right|^{2}\right. \\
& \left.+\operatorname{Im}\left(\frac{q_{1}}{\mu_{1}}\right) \operatorname{Im}\left(\frac{\tilde{q}_{2}}{\tilde{\mu}_{2}}\right)(1+\beta)\left|Q_{\varepsilon}\right|^{2}\right] \\
& +\frac{4|q|^{2}}{|Q|^{2}}\left[\operatorname{Im}\left(\frac{q_{1}}{\varepsilon_{1}}\right) \operatorname{Im}\left(\frac{\tilde{q}_{2}}{\tilde{\mu}_{2}}\right)|\beta|\left|Q_{\mu \varepsilon}\right|^{2}\right. \\
& \left.+\operatorname{Im}\left(\frac{q_{1}}{\mu_{1}}\right) \operatorname{Im}\left(\frac{\tilde{q}_{2}}{\tilde{\varepsilon}_{2}}\right)|\beta|\left|Q_{\varepsilon \mu}\right|^{2}\right],
\end{aligned}
$$

где $q_{1}=\left(k^{2}-(\omega / c)^{2} \varepsilon_{1} \mu_{1}\right)^{1 / 2}, q_{2}=\left(k^{2}-(\omega / c)^{2} \varepsilon_{2} \mu_{2}\right)^{1 / 2}$, $q=\left(k^{2}-(\omega / c)^{2}\right)^{1 / 2}$. Комплексные значения квадратных корней выбираются из условия $\operatorname{Re} q_{1,2}>0$, а параметр $\beta$ определяется соотношением

$$
\beta=\frac{\gamma^{2} u^{2} q^{2} k_{\perp}^{2}}{k^{2} \tilde{k}^{2}}
$$

В (5), кроме того, $k_{\perp}^{2}=\left[\mathbf{k}-\frac{\mathbf{u}(\mathbf{k u})}{u^{2}}\right]^{2}$ и $\quad \tilde{\mathbf{k}}=\mathbf{k}$ $+(\gamma-1) \frac{\mathbf{u}(\mathbf{k u})}{u^{2}}-\gamma k \mathbf{u}(\tilde{\mathbf{k}}-$ волновой вектор в системе покоя пластины 2), а значок „тильда“ означает, что соответствующие величины, зависящие от $\omega$ и $k$ берутся при $\tilde{\omega}$ и $\tilde{k}$. Величины $Q_{\varepsilon}, Q_{\mu}, Q_{\varepsilon \mu}, Q_{\mu \varepsilon}, Q$ в (4) имеют вид $(a-$ ширина щели)

$$
\begin{aligned}
Q_{\varepsilon}= & \left(q+q_{1} / \varepsilon_{1}\right)\left(q+\tilde{q}_{2} / \tilde{\varepsilon}_{2}\right) \exp (q a) \\
& -\left(q-q_{1} / \varepsilon_{1}\right)\left(q-\tilde{q}_{2} / \tilde{\varepsilon}_{2}\right) \exp (-q a), \\
Q_{\mu}= & \left(q+q_{1} / \mu_{1}\right)\left(q+\tilde{q}_{2} / \tilde{\mu}_{2}\right) \exp (q a) \\
& -\left(q-q_{1} / \mu_{1}\right)\left(q-\tilde{q}_{2} / \tilde{\mu}_{2}\right) \exp (-q a), \\
Q_{\varepsilon \mu}= & \left(q+q_{1} / \varepsilon_{1}\right)\left(q+\tilde{q}_{2} / \tilde{\mu}_{2}\right) \exp (q a) \\
& -\left(q-q_{1} / \varepsilon_{1}\right)\left(q-\tilde{q}_{2} / \tilde{\mu}_{2}\right) \exp (-q a), \\
Q_{\mu \varepsilon}= & \left(q+q_{1} / \mu_{1}\right)\left(q+\tilde{q}_{2} / \tilde{\varepsilon}_{2}\right) \exp (q a) \\
& -\left(q-q_{1} / \mu_{1}\right)\left(q-\tilde{q}_{2} / \tilde{\varepsilon}_{2}\right) \exp (-q a), \\
Q=Q_{\varepsilon} Q_{\mu}- & 4 \beta k^{2} \tilde{\mu}_{2}\left(1-\left(\varepsilon_{1} \mu_{1}\right)^{-1}\right)\left(1-\left(\tilde{\varepsilon}_{2} \tilde{\mu}_{2}\right)^{-1}\right) .
\end{aligned}
$$

Мы сохранили все обозначения, использовавшиеся в [5], с единственной заменой $\kappa, \kappa_{1} \kappa_{2} \rightarrow \mathbf{k}, k_{1}, k_{2}$. 


\section{3. Преобразование общей формулы при $u=V / c \ll 1$}

В случае $u=V / c \ll 1$ мы имеем $\beta=0, \tilde{\mathbf{k}}=\mathbf{k}$, и с учетом (10) формула (4) сводится к

$$
\begin{aligned}
M= & 4|q|^{2}\left[\operatorname{Im}\left(\frac{q_{1}}{\varepsilon_{1}}\right) \operatorname{Im}\left(\frac{\tilde{q}_{2}}{\tilde{\varepsilon}_{2}}\right)\left|Q_{\varepsilon}\right|^{-2}\right. \\
& \left.+\operatorname{Im}\left(\frac{q_{1}}{\mu_{1}}\right) \operatorname{Im}\left(\frac{\tilde{q}_{2}}{\tilde{\mu}_{2}}\right)\left|Q_{\mu}\right|^{-2}\right] .
\end{aligned}
$$

Используя (11) и тождество

$$
4 \operatorname{Im}\left(\frac{q_{1}}{\varepsilon_{1}}\right) \operatorname{Im}\left(\frac{\tilde{q}_{2}}{\tilde{\varepsilon}_{2}}\right) \equiv-\left(\frac{q_{1}}{\varepsilon_{1}}-\frac{q_{1}^{*}}{\varepsilon_{1}^{*}}\right)\left(\frac{\tilde{q}_{2}}{\tilde{\varepsilon}_{2}}-\frac{\tilde{q}_{2}^{*}}{\tilde{\varepsilon}_{2}^{*}}\right),
$$

а также аналогичное тождество с перестановками $\varepsilon_{1} \leftrightarrow \mu_{1}, \quad \tilde{\varepsilon}_{2} \leftrightarrow \tilde{\mu}_{2}$ формула (2) для $P_{1}$ сводится (при $V=0)$ к формуле (6) в [3] для спектральной плотности потока энергии теплового поля (поскольку $\tilde{q}_{2}=q_{2}$, $\left.\tilde{\varepsilon}_{2}=\varepsilon_{2}\right)$. В этом случае $P_{1}=-P_{2},\left|P_{1}\right|=\left|P_{2}\right|$, а разный знак у этих величин обусловлен разным направлением потока тепловой энергии относительно пластин.

При $V \neq 0$, используя (6), выражение для $\left|Q_{\varepsilon}\right|^{-2}$ приводится к виду

$$
\begin{aligned}
& \left|Q_{\varepsilon}\right|^{-2}=\left|\varepsilon_{1} q+q_{1}\right|^{-2}\left|q \tilde{\varepsilon}_{2}+\tilde{q}_{2}\right|^{-2} \\
& \quad \times\left|1-\Delta_{1 e} \tilde{\Delta}_{2 e} \exp (-2 q a)\right|^{-2}\left|\varepsilon_{1}\right|^{2}\left|\tilde{\varepsilon}_{2}\right|^{2}|\exp (-2 q a)|,
\end{aligned}
$$

где

$$
\Delta_{1 e}=\frac{\varepsilon_{1} q-q_{1}}{\varepsilon_{1} q+q_{1}}, \quad \tilde{\Delta}_{2 e}=\frac{\tilde{\varepsilon}_{2} q-\tilde{q}_{2}}{\tilde{\varepsilon}_{2} q-\tilde{q}_{2}} .
$$

Для $\left|Q_{\mu}\right|^{-2}$ получается такое же выражение, как и для $\left|Q_{\varepsilon}\right|^{-2}$ с учетом очевидных замен $\varepsilon_{1} \leftrightarrow \mu_{1}, \tilde{\varepsilon}_{2} \leftrightarrow \tilde{\mu}_{2}$, $\Delta_{1 e} \rightarrow \Delta_{1 m}, \tilde{\Delta}_{2 e} \rightarrow \tilde{\Delta}_{2 m}$.

Интегралы в (2) и (3) содержат вклады от неоднородных волн $(k>\omega / c)$ и от бегущих волн $k \leq \omega / c$. При $k>\omega / c$ имеем $q=|q|,|\exp (-2 q a)|=\exp (-2 q a)$, и (12) принимает вид

$$
\begin{aligned}
\left(\frac{q_{1}}{\varepsilon_{1}}\right. & \left.-\frac{q_{1}^{*}}{\varepsilon_{1}^{*}}\right)\left(\frac{\tilde{q}_{2}}{\tilde{\varepsilon}_{2}}-\frac{\tilde{q}_{2}^{*}}{\tilde{\varepsilon}_{2}^{*}}\right) \\
& =-\frac{\operatorname{Im} \Delta_{1 e} \operatorname{Im} \tilde{\Delta}_{2 e}}{|q|^{2}\left|\varepsilon_{1}\right|^{2}\left|\tilde{\varepsilon}_{2}\right|^{2}}\left|\varepsilon_{1} q+q_{1}\right|^{2}\left|\tilde{\varepsilon}_{2} q+\tilde{q}_{2}\right|^{2} .
\end{aligned}
$$

При $\quad k \leq \omega / c \quad$ соответственно $q=-i|q|$, $|\exp (-2 q a)|=1$ и

$$
\begin{aligned}
& \left(\frac{q_{1}}{\varepsilon_{1}}-\frac{q_{1}^{*}}{\varepsilon_{1}^{*}}\right)\left(\frac{\tilde{q}_{2}}{\tilde{\varepsilon}_{2}}-\frac{\tilde{q}_{2}^{*}}{\tilde{\varepsilon}_{2}^{*}}\right) \\
& =-\frac{\left(1-\left|\Delta_{1 e}\right|^{2}\right)\left(1-\left|\tilde{\Delta}_{2 e}\right|^{2}\right)}{4|q|^{2}\left|\varepsilon_{1}\right|^{2}\left|\tilde{\varepsilon}_{2}\right|^{2}}\left|\varepsilon_{1} q+q_{1}\right|^{2}\left|\tilde{\varepsilon}_{2} q+\tilde{q}_{2}\right|^{2} .
\end{aligned}
$$

Аналогичные (14), (15) тождества получаются путем замены $\varepsilon_{1} \leftrightarrow \mu_{1}, \tilde{\varepsilon}_{2} \leftrightarrow \tilde{\mu}_{2}, \Delta_{1 e} \rightarrow \Delta_{1 m}, \tilde{\Delta}_{2 e} \rightarrow \tilde{\Delta}_{2 m}$.
Подставляя (11)-(15) в (1)-(3), и принимая во внимание аналитические свойства функции $M(\omega, \mathbf{k}, \mathbf{u})[5]$

$$
\begin{aligned}
& M(\omega, \mathbf{k}, \mathbf{u})>0, \quad \omega \tilde{\omega}>0, \\
& M(\omega, \mathbf{k}, \mathbf{u})>0, \quad \omega \tilde{\omega}<0,
\end{aligned}
$$

получим следующее выражение для тангенциальной силы, действующей на движущуюся пластину 2 в лабораторной системе отсчета (отрицательные значения $F_{x}$ соответствуют диссипативной силе трения)

$$
\begin{aligned}
& F_{x}=-\frac{\hbar}{4 \pi^{3}} \int_{0}^{\infty} d \omega \int_{k>\omega / c} d^{2} k k_{x} \exp (-2 q a) \\
& \times \operatorname{Im} \Delta_{1 e} \operatorname{Im} \tilde{\Delta}_{2 e}\left|D_{e}\right|^{-2}\left[\operatorname{coth}\left(\hbar \tilde{\omega} / 2 T_{2}\right)-\operatorname{coth}\left(\hbar \omega / 2 T_{1}\right)\right] \\
& -\frac{\hbar}{16 \pi^{3}} \int_{0}^{\infty} d \omega \int_{k \leq \omega / c} d^{2} k k_{x}\left(1-\left|\Delta_{1 e}\right|^{2}\right)\left(1-\left|\tilde{\Delta}_{2 e}\right|^{2}\right)\left|D_{e}\right|^{-2} \\
& \times\left[\operatorname{coth}\left(\hbar \tilde{\omega} / 2 T_{2}\right)-\operatorname{coth}\left(\hbar \omega / 2 T_{1}\right)\right]+(e \leftrightarrow m),
\end{aligned}
$$

где $\left|D_{e}\right|=\left|1-\Delta_{1 e} \tilde{\Delta}_{2 e} \exp (-2 q a)\right|$, а слагаемые $(e \leftrightarrow m)$ определяются такими же интегралами с заменами $\varepsilon_{1} \leftrightarrow \mu_{1}, \quad \tilde{\varepsilon}_{2} \leftrightarrow \tilde{\mu}_{2}, \Delta_{1 e} \rightarrow \Delta_{1 m}, \tilde{\Delta}_{2 e} \rightarrow \tilde{\Delta}_{2 m}$. Подчеркнем еще раз, что в рассматриваемом случае $\tilde{\omega}=\omega-k_{x} V$.

Формула (17) полностью включает все результаты других авторов $[10-13,16-20]$ при $u=V / c \ll 1$, полученные в незапаздывающем и запаздывающем пределе. В частности, в пределе $T_{1} \rightarrow 0, T_{2} \rightarrow 0$ из (17) вытекает формула для силы квантового трения $[13,17]$

$$
\begin{aligned}
F_{x}= & \frac{\hbar}{4 \pi^{3}} \int_{-\infty}^{\infty} d k_{y} \int_{0}^{\infty} d k_{x} k_{x} \int_{0}^{k_{x} V} d \omega \exp (-2 k a) \operatorname{Im} \Delta_{1 e} \\
& \times \operatorname{Im} \tilde{\Delta}_{2 e}\left|D_{e}\right|^{-2}+(e \leftrightarrow m) .
\end{aligned}
$$

В свою очередь, в случае покоящихся пластин $(V=0)$, формула для результирующего потока энергии теплового поля $P_{1}$ от первой пластины (для определенности), принимая во внимание (11)-(16), сводится к [8-10]

$$
\begin{aligned}
& P_{1}=\frac{\hbar}{2 \pi^{3}} \int_{0}^{\infty} d \omega \omega \int_{k>\omega / c} d^{2} k \exp (-2 q a) \\
& \times \operatorname{Im} \Delta_{1 e} \operatorname{Im} \Delta_{2 e}\left|D_{e}\right|^{-2}\left[n_{1}(\omega)-n_{2}(\omega)\right] \\
& +\frac{\hbar}{8 \pi^{3}} \int_{0}^{\infty} d \omega \omega \int_{k \leq \omega / c} d^{2} k\left(1-\left|\Delta_{1 e}\right|^{2}\right)\left(1-\left|\Delta_{2 e}\right|^{2}\right)\left|D_{e}\right|^{-2} \\
& \times\left[n_{1}(\omega)-n_{2}(\omega)\right]+(e \leftrightarrow m),
\end{aligned}
$$

где $n_{i}(\omega)=1 /\left(\exp \left(\hbar \omega / T_{i}\right)-1\right), i=1,2$. 
Другое, более компактное представление для силы трения, получается из (17) с учетом тождеств (13)-(15)

$$
\begin{aligned}
& F_{x}=-\frac{\hbar}{4 \pi^{3}} \int_{0}^{\infty} d \omega \int d^{2} k k_{x}\left[\operatorname{Im}\left(\frac{q_{1}}{\varepsilon_{1}}\right) \operatorname{Im}\left(\frac{\tilde{q}_{2}}{\tilde{\varepsilon}_{2}}\right) \frac{|q|^{2}}{\left|Q_{\varepsilon}\right|^{2}}\right. \\
& \left.+\operatorname{Im}\left(\frac{q_{1}}{\mu_{1}}\right) \operatorname{Im}\left(\frac{\tilde{q}_{2}}{\tilde{\mu}_{2}}\right) \frac{|q|^{2}}{\left|Q_{\mu}\right|^{2}}\right]\left[\operatorname{coth}\left(\frac{\hbar \tilde{\omega}}{2 T_{2}}\right)-\operatorname{coth}\left(\frac{\hbar \omega}{2 T_{1}}\right)\right] .
\end{aligned}
$$

Заметим, что внутренний интеграл в (20) включает суммарный вклад неоднородных и однородных волн, a также магнитных и электрических составляющих. Формула (20) оказывается более удобной для численных расчетов силы трения металлических пластин. Таким же образом, общее выражение для $P_{1}$ (в случае $0<V / c \ll 1)$ можно записать в виде

$$
\begin{aligned}
& P_{1}=\frac{\hbar}{4 \pi^{3}} \int_{0}^{\infty} d \omega \omega \int d^{2} k\left[\operatorname{Im}\left(\frac{q_{1}}{\varepsilon_{1}}\right) \operatorname{Im}\left(\frac{\tilde{q}_{2}}{\tilde{\varepsilon}_{2}}\right) \frac{|q|^{2}}{\left|Q_{\varepsilon}\right|^{2}}\right. \\
& \left.+\operatorname{Im}\left(\frac{q_{1}}{\mu_{1}}\right) \operatorname{Im}\left(\frac{\tilde{q}_{2}}{\tilde{\mu}_{2}}\right) \frac{|q|^{2}}{\left|Q_{\mu}\right|^{2}}\right]\left[\operatorname{coth}\left(\frac{\hbar \tilde{\omega}}{2 T_{2}}\right)-\operatorname{coth}\left(\frac{\hbar \omega}{2 T_{1}}\right)\right] .
\end{aligned}
$$

Вполне очевидно, что (21) сводится к (19) при $V=0$. Таким образом, фундаментальные результаты теории [35] (формулы (1) - (10)) полностью включают все результаты других авторов для скорости теплообмена и силы трения в конфигурации двух произвольных полубесконечных сред (толстых пластин) при их относительном движении.

\section{4. Некоторые следствия и частные случаи}

В дополнение к формулам (17), (18) и (20), вытекающим из (1)-(3), целесообразно рассмотреть другие известные предельные случаи.

При малых скоростях скольжения и достаточно высокой температуре $T_{1}=T_{2}=T, \quad k_{x} V / \omega_{w} \ll 1$ $\left(\omega_{w}=T / \hbar-\right.$ частота Вина), тепловой фактор в (17) и (20) принимает вид

$$
\begin{gathered}
\operatorname{coth}\left(\frac{\hbar \tilde{\omega}}{2 T_{2}}\right)-\operatorname{coth}\left(\frac{\hbar \omega}{2 T_{1}}\right) \approx-2 k_{x} V \frac{d n}{d \omega}, \\
n(\omega)=\frac{1}{\exp \left(\omega / \omega_{w}\right)-1} .
\end{gathered}
$$

С другой стороны, в разложении первого порядка по скорости $V$, величины со значком „тильда“ в (17) и (20) будут содержать не зависящие от скорости члены, и пропорциональные скорости члены. Поэтому, учитывая вид (20) и (22), приближение первого порядка по скорости для силы трения принимает вид

$$
\begin{aligned}
F_{x}= & \frac{\hbar V}{2 \pi^{2}} \int_{0}^{\infty} d \omega \frac{d n(\omega)}{d \omega} \int_{0}^{\infty} d k k^{3}\left[\operatorname{Im}\left(\frac{q_{1}}{\varepsilon_{1}}\right) \operatorname{Im}\left(\frac{q_{2}}{\varepsilon_{2}}\right) \frac{|q|^{2}}{\left|Q_{\varepsilon}\right|^{2}}\right. \\
& \left.+\operatorname{Im}\left(\frac{q_{1}}{\mu_{1}}\right) \operatorname{Im}\left(\frac{q_{2}}{\mu_{2}}\right) \frac{|q|^{2}}{\left|Q_{\mu}\right|^{2}}\right] .
\end{aligned}
$$

Следует подчеркнуть, что все величины, входящие в $\left|Q_{\varepsilon}\right|^{-2}$ и $\left|Q_{\mu}\right|^{-2}$ (см. (13)) в этом случае не содержат значка „тильда“. В свою очередь, формула (17) запишется в виде

$$
\begin{aligned}
& F_{x}=\frac{\hbar V}{2 \pi^{3}} \int_{0}^{\infty} d \omega \frac{d n(\omega)}{d \omega} \int_{k>\omega / c} d^{2} k k_{x}^{2} \exp (-2 q a) \\
& \times \operatorname{Im} \Delta_{1 e} \operatorname{Im} \Delta_{2 e}\left|D_{e}\right|^{-2}+\frac{\hbar V}{8 \pi^{3}} \int_{0}^{\infty} d \omega \frac{d n(\omega)}{d \omega} \\
& \times \int_{k \leq \omega / c} d^{2} k k_{x}^{2}\left(1-\left|\Delta_{1 e}\right|^{2}\right)\left(1-\left|\Delta_{2 e}\right|^{2}\right)\left|D_{e}\right|^{-2}(e \leftrightarrow m) .
\end{aligned}
$$

В предельном случае абсолютно черных тел, $\varepsilon_{1}=\varepsilon_{2}=\mu_{1}=\mu_{2}=1$, величина подынтегрального выражения в квадратных скобках (23) равна (-2) при $k<\omega / c$ и нулю в противном случае. Последующее вычисление интеграла приводит к результату

$$
F_{x}=-\frac{\pi^{2}}{60} \frac{\hbar V}{c^{4}}\left(\frac{T}{\hbar}\right)^{4} .
$$

Такая же формула получается из (24), поскольку $\Delta_{1 e}=\Delta_{2 e}=\Delta_{1 m}=\Delta_{2 m}=0$. При $T=300 \mathrm{~K}$ и $V=1 \mathrm{~m} / \mathrm{s}$ из формулы (25) следует оценка $F \sim 5 \cdot 10^{-15} \mathrm{~N} / \mathrm{m}^{2}$, не зависящая от ширины щели.

В частном случае $\omega_{w} a / c \ll 1$ (т.е. $a \ll 7.6 \mu$ m при $T=300 \mathrm{~K})$, используя приближение $\left(k^{2}-\omega^{2} / c^{2}\right)^{1 / 2} \approx k$, получим $\Delta_{i e} \approx\left(\varepsilon_{i}-1\right) /\left(\varepsilon_{i}+1\right)$ и $\Delta_{i m} \approx \frac{\omega^{2}\left(\varepsilon_{i}-1\right)}{4 k^{2} c^{2}}(i=1,2)$. Тогда, для диэлектриков и плохих проводников, $\Delta_{i m} \approx 0$, a (24) преобразуется к виду

$$
\begin{aligned}
F_{x} \approx & \frac{\hbar V}{2 \pi^{3}} \int_{0}^{\infty} \frac{d n(\omega)}{d \omega} d \omega \operatorname{Im}\left(\frac{\varepsilon_{1}-1}{\varepsilon_{1}+1}\right) \operatorname{Im}\left(\frac{\varepsilon_{2}-1}{\varepsilon_{2}+1}\right) \\
& \times \int_{0}^{\infty} d k k^{3} \exp (-2 k a)\left|D_{e}\right|^{-2},
\end{aligned}
$$

где

$$
D_{e}=1-\left(\frac{\varepsilon_{1}-1}{\varepsilon_{1}+1}\right)\left(\frac{\varepsilon_{2}-1}{\varepsilon_{2}+1}\right) \exp (-2 k a) .
$$

Для контакта проводников с диэлектрическими проницаемостями $\varepsilon_{1,2}=1+i 4 \sigma_{1,2} / \omega$, при условии $\omega_{w} / 2 \pi \sigma \ll 1$ 
будем иметь $\left|D_{e}\right| \approx 1$, и из (26) следует

$$
F_{x} \approx-\frac{1}{64 \pi^{2}} \frac{(T / \hbar)^{2}}{\sigma_{1} \sigma_{2}} \frac{\hbar V}{a^{4}} .
$$

При $T=300 \mathrm{~K}, V=1 \mathrm{~m} / \mathrm{s}, \sigma_{1}=\sigma_{2}=10^{14} \mathrm{~s}^{-1}$ (графит) и $a=10 \mathrm{~nm}$ формула (28) дает $F \sim 2.6 \cdot 10^{-6} \mathrm{~N} / \mathrm{m}^{2}$. Причем следует отметить, что величина $F_{x}$ уменьшается с увеличением проводимости материалов.

Для хорошо проводящих металлов, однако, приведенные выше оценки некорректны, и требуется более точный расчет. В [5], в частности, использовалось импедансное приближение с фактором $\left(k^{2}-\varepsilon_{i} \mu_{i} \omega^{2}\right)^{1 / 2} \approx i\left(\varepsilon_{i} \mu_{i}\right)^{1 / 2} \omega / c=i \varepsilon_{i} \xi_{i}$ (где $\xi_{i}$ - импеданс). Это в итоге привело к функциональной зависимости $F \sim V T^{7 / 2} / \sigma^{1 / 2} a$ при малой ширине щели для немагнитных металлов с $\sigma_{1}=\sigma_{2}=\sigma$ и $\mu_{1}=\mu_{2}=1$. Соответствующая численная оценка, полученная в [5], составляла $\sim 3 \cdot 10^{-14} \mathrm{~N} / \mathrm{m}^{2}$, при $\sigma=5 \cdot 10^{17} \mathrm{~s}^{-1}$ и тех же других условиях, предполагавшихся выше. Однако, как мы покажем далее, численный расчет с использованием точного фактора $\left(k^{2}-\varepsilon_{i} \mu_{i} \omega^{2} / c^{2}\right)^{1 / 2}$ приводит к значительно более высоким оценкам силы трения.

\section{5. Случай хорошо проводящих немагнитных металлов}

Преобразуем формулу (20) к форме, удобной для последующего численного расчета. Сначала рассмотрим случай идентичных металлов $\sigma_{1}=\sigma_{2}=\sigma, \mu_{1}=\mu_{2}=1$ с диэлектрической функцией $\varepsilon(\omega)=i 4 \pi \sigma / \omega$, аналогично [5]. Тогда будем иметь

$$
\begin{gathered}
\left(k^{2}-\varepsilon \omega^{2} / c^{2}\right)^{1 / 2}=\left(k^{4}+\frac{(4 \pi \sigma \omega)^{2}}{c^{4}}\right)^{1 / 4} \exp (i \phi), \\
\phi=-0.5 \arctan \left(\frac{4 \pi \sigma \omega}{k^{2} c^{2}}\right) .
\end{gathered}
$$

Чтобы найти вклад от неоднородных мод $k>\omega / c$, введем новые переменные $\omega=\omega_{w} x$ и $k^{2}=\left(y^{2}+\lambda_{a}^{2} x^{2}\right) / a^{2}$ $\left(\lambda_{a}=\omega_{w} a / c\right), k d k=y d y / a^{2}$. Наиболее значительный вклад в интеграл (23) дает второй член в квадратных скобках. Соответствующий ему внутренний интеграл приводится к виду

$$
\begin{aligned}
& \int_{\omega / c}^{\infty} d k k^{3} \operatorname{Im}\left(\frac{q_{1}}{\mu_{1}}\right) \operatorname{Im}\left(\frac{q_{2}}{\mu_{2}}\right) \frac{|q|^{2}}{\left|Q_{\mu}\right|^{2}} \\
& =\frac{1}{4 a^{4}} \int_{0}^{\infty} d y y^{3}\left(y^{2}+\lambda_{a}^{2} x^{2}\right) U_{1}^{2}\left|Q_{\mu 1}\right|^{-2} \sin ^{2}\left(\phi_{1}\right) \equiv \frac{1}{a^{4}} I_{\mu 1}, \\
& U_{1}=\left[\left(y^{2}+\lambda_{a}^{2} x^{2}\right)^{2}+\lambda^{2} x^{2}\right]^{1 / 4}, \quad \lambda=\frac{4 \pi \sigma \omega_{w} a^{2}}{c^{2}},
\end{aligned}
$$

$$
\begin{aligned}
\left|Q_{\mu 1}\right|^{2}=\mid\left(y^{2}+U_{1}^{2} \exp \left(2 i \phi_{1}\right)\right) \sinh (y) \\
+\left.2 U_{1} \exp \left(i \phi_{1}\right) \cosh (y)\right|^{2}, \\
\phi_{1}=-0.5 \arctan \left(\frac{\lambda x}{y^{2}+\lambda_{a}^{2} x^{2}}\right) .
\end{aligned}
$$

Вклад от однородных мод $k<\omega / c$ для этого слагаемого в (23) находится с помощью замены переменных $\omega=\omega_{w} x$ и $k^{2}=\frac{\omega_{w}^{2}}{c^{2}}\left(x^{2}-y^{2}\right), k d k=-y d y \omega_{w}^{2} / c^{2}$. Тогда получим

$$
\begin{gathered}
\int_{0}^{\infty} d k k^{3} \operatorname{Im}\left(\frac{q_{1}}{\mu_{1}}\right) \operatorname{Im}\left(\frac{q_{2}}{\mu_{2}}\right) \frac{|q|^{2}}{\left|Q_{\mu}\right|^{2}}=\frac{\omega_{w}^{4}}{4 c^{4}} \\
\times \int_{0}^{x} d y y^{3}\left(x^{2}-y^{2}\right) U_{2}^{2}\left|Q_{\mu 2}\right|^{-2} \sin ^{2}\left(\phi_{2}\right) \equiv \frac{\omega_{w}^{4}}{c^{4}} I_{\mu 2}, \\
U_{2}=\left(\left(x^{2}-y^{2}\right)^{2}+\tilde{\lambda}^{2} x^{2}\right)^{1 / 4}, \quad \tilde{\lambda}=\frac{4 \pi \sigma}{\omega_{w}} \\
\left|Q_{\mu 2}\right|^{2}=\mid\left(y^{2}+U_{2}^{2} \exp \left(2 i \phi_{2}\right)\right) \sin \left(\lambda_{a} y\right) \\
-\left.2 y U_{2} \exp \left(i \phi_{2}\right) \cos \left(\lambda_{a} y\right)\right|^{2} \\
\phi_{2}=-0.5 \arctan \left(\frac{\tilde{\lambda} x}{y^{2}-y^{2}}\right) .
\end{gathered}
$$

Выполняя такие же замены переменных $\omega, k$ в интегралах, соответствующих первому слагаемому в квадратных скобках (23), будем иметь:

а) вклад неоднородных мод

$$
\begin{gathered}
\int_{\omega / c}^{\infty} d k k^{3} \operatorname{Im}\left(\frac{q_{1}}{\varepsilon_{1}}\right) \operatorname{Im}\left(\frac{q_{2}}{\varepsilon_{2}}\right) \frac{|q|^{2}}{\left|Q_{\varepsilon}\right|^{2}}=\frac{1}{4 a^{4}} \int_{0}^{\infty} d y y^{3} \\
\times\left(y^{2}+\lambda_{a}^{2} x^{2}\right) x^{2} \tilde{\lambda}^{-2} U_{1}^{2}\left|Q_{\varepsilon 1}\right|^{-2} \cos ^{2}\left(\phi_{1}\right) \equiv \frac{1}{a^{4}} I_{\varepsilon 1}, \\
\left|Q_{\varepsilon 1}\right|^{2}=\mid\left(y^{2}-x^{2} \tilde{\lambda}^{-2} U_{1}^{2} \exp \left(2 i \phi_{1}\right)\right) \sinh (y) \\
-\left.i 2 x \tilde{\lambda}^{-1} y U_{1} \exp \left(i \phi_{1}\right) \cosh (y)\right|^{2} .
\end{gathered}
$$

б) вклад однородных мод

$$
\begin{gathered}
\int_{0}^{\omega / c} d k k^{3} \operatorname{Im}\left(\frac{q_{1}}{\varepsilon_{1}}\right) \operatorname{Im}\left(\frac{q_{2}}{\varepsilon_{2}}\right) \frac{|q|^{2}}{\left|Q_{\varepsilon}\right|^{2}}=\frac{\omega_{w}^{4}}{4 c^{4}} \int_{0}^{x} d y y^{3} \\
\times x^{2} \tilde{\lambda}^{-2}\left(x^{2}-y^{2}\right) U_{2}\left|Q_{\varepsilon 2}\right|^{-2} \cos 2\left(\phi_{2}\right) \equiv \frac{\omega_{w}^{4}}{c^{4}} I_{\varepsilon 2}, \\
\left|Q_{\varepsilon 2}\right|^{2}=\mid i\left(y^{2}+x^{2} \tilde{\lambda}^{-2} U_{2}^{2} \exp \left(2 i \phi_{2}\right)\right) \sin \left(\lambda_{a} y\right) \\
-\left.2 x \tilde{\lambda}^{-1} y U_{2} \exp \left(i \phi_{2}\right) \cos \left(\lambda_{a} y\right)\right|^{2} .
\end{gathered}
$$




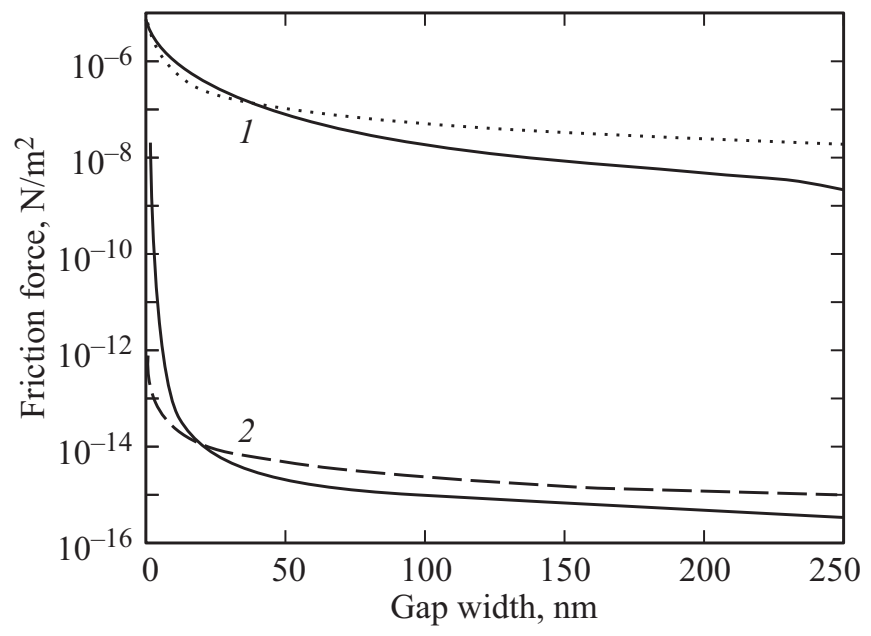

Рис. 2. Сила трения (42) (сплошная линия) и (43) (штриховая линия). Пунктирная кривая - аппроксимация $F \sim 1 / a$, сплошные линии 1 и 2 соответствуют слагаемым $I_{\mu 1}$ и $I_{\varepsilon 1}$. Используемые параметры: $\sigma_{1}=\sigma_{2}=5 \cdot 10^{17} \mathrm{~s}^{-1}, T=300 \mathrm{~K}$, $V=1 \mathrm{~m} / \mathrm{s}$.

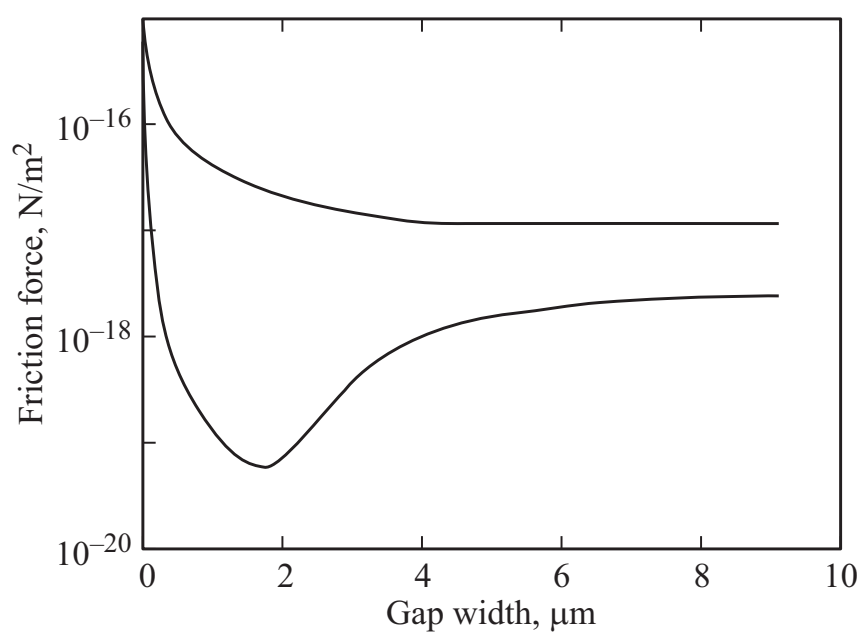

Рис. 3. Вклады в силу трения (42) от однородных мод, связанные со слагаемыми $I_{\varepsilon 2}$ (верхняя кривая) и $I_{\mu 2}$ (нижняя кривая), $\sigma_{1}=\sigma_{2}=5 \cdot 10^{17} \mathrm{~s}^{-1}, T=300 \mathrm{~K}, V=1 \mathrm{~m} / \mathrm{s}$.

Далее, подставляя (30), (34), (38), (40) в (23), получим

$$
\begin{aligned}
F_{x}= & -\frac{\hbar V}{2 \pi^{2} a^{4}} \int_{0}^{\infty} d x \frac{\exp (x)}{(\exp (x)-1)^{2}} \\
& \times\left(I_{\mu 1}+I_{\mu 2}\left(\omega_{w} a / c\right)^{4}+I_{\varepsilon 1}+I_{\varepsilon 2}\left(\omega_{w} a / c\right)^{4}\right) .
\end{aligned}
$$

Как вытекает из (34), (40) и (42), зависимость силы $F_{x}$ от ширины щели $a$ для однородных мод значительно слабее, чем для неоднородных, поэтому при малой величине $a$ вкладом неоднородных мод можно пренебречь. В свою очередь, вклады от неоднородных мод будут незначительны при большой ширине щели.

Значения сил $F_{x}$ на рис. 2,3 , рассчитанные в низкочастотном приближении Друде с фиксированной прово- димостью $\sigma$, здесь и далее приведены с положительным знаком. Сплошные кривые 1 и 2 на рис. 2 соответствуют слагаемым $I_{\mu 1}$ и $I_{\mu 2}$ в (42) (т. е. вкладам неоднородных волн). Штриховая кривая показывает импедансное приближение [5], в данном случае

$$
F_{p}=-\frac{105 \xi(7 / 2)}{2^{17 / 2} \cdot \pi} \frac{T^{7 / 2}}{\sigma^{1 / 2} \hbar^{5 / 2} c^{3}} \frac{V}{a},
$$

где $\zeta(7 / 2)=1.127$ - дзета-функция Римана. Вклады от однородных мод в (42), не показанные на рис. 2, пренебрежимо малы и показаны отдельно на рис. 3. Как следует из рис. 2, сила трения металлических пластин в области нанометровых расстояний оказывается в $10^{7}$ раз выше, чем первоначальная оценка в [5]. В области расстояний $1 \leq a \leq 30 \mathrm{~nm}$ сила трения убывает с расстоянием несколько медленнее чем $1 / a$, но далее наклон зависимости становится ближе к $1 / a^{2}$. Возрастание силы трения при малой ширине зазора физически обусловлено высокими значениями коэффициентов отражения металлических пластин, в результате чего электромагнитные волны, приобретающие допплеровский сдвиг из-за относительного движения пластин, многократно отражаются от них, что, в итоге, и приводит к увеличению силы трения. При этом величина силы трения на 7-8 порядков величины выше, чем в случае абсолютно черных пластин. Кроме того, доминирующий вклад в трение связан с магнитными слагаемыми (42), обусловленными вихревыми токами.

Весьма интересно рассмотреть температурную зависимость $F_{x}$, связанную с изменением времени релаксации и проводимости металла при понижении температуры, когда величина $1 / \tau$ имеет порядок виновской частоты $\omega_{w}$. Для этой цели мы провели расчеты с использованием функции Друде общего вида

$$
\begin{aligned}
\varepsilon_{D}(\omega) & =1-\frac{\omega_{p}^{2}}{\omega(\omega+i / \tau)}=1-\frac{\omega_{p}^{2} \tau^{2}}{\omega \tau(\omega \tau+i)} \\
& =1-\frac{\kappa_{p}^{2}\left(\kappa_{w} x-i\right)}{\kappa_{w} x\left(1+\kappa_{w}^{2} x^{2}\right)}
\end{aligned}
$$

Здесь использована параметризация $\kappa_{p}=\omega_{p} \tau$, $\kappa_{w}=\omega_{w} \tau, x=\omega / \omega_{w}$. Зависимость $\tau(T)$ описывалась формулой Блоха-Грюнайзена [20]

$$
\tau^{-1}=0.0847(T / \theta)^{5} \int_{0}^{T / \theta} \frac{x^{5} \exp (x)}{(\exp (x)-1)^{2}} d x(\mathrm{eV}),
$$

где $\theta$ - температура Дебая. В расчетах принимались значения $\omega_{p}=9.03 \mathrm{eV}$ и $\theta=175 \mathrm{~K}$, соответствующие золоту. При вычислении интегралов в (30), (34), (38) и (40) с использованием (44), общая структура формул (30)-(42) не изменяется. Необходимые модификации формул (31), (33), (35) и (37)-(40) приведены в Приложении. 
Результаты расчетов силы $F_{x}$ в зависимости от температуры и расстояния показаны на рис. 4 , а на рис. 5 - в зависимости от расстояния и температуры. Кривые $1-3$ на рис. 4 соответствуют расстояниям 10, 100, $1000 \mathrm{~nm}$, а кривые $1-3$ на рис. 5 - температурам 5, 100 и $300 \mathrm{~K}$. Как видно из рис. 4 , сила трения резко возрастает при $T<50 \mathrm{~K}$. Вывод о том, что оценка, сделанная в [5] (при $T=300 \mathrm{~K})$, занижена в $10^{7}$ раз, остается в силе. Из рис. 4,5 следует также ошибочность утверждения о том, что сила трения максимальна в случае плохих проводников типа графита $[10,13]$. Зависимости от ширины щели для различных вкладов в силу $F_{x}$ при $T=300 \mathrm{~K}$ демонстрирует рис. 6. Магнитные вклады $I_{\mu 1}, I_{\mu 2}$, от неоднородных и однородных волн показаны толстыми кривыми 1,2 , а электрические вклады $I_{\varepsilon 1}, I_{\varepsilon 2}$, от неод-

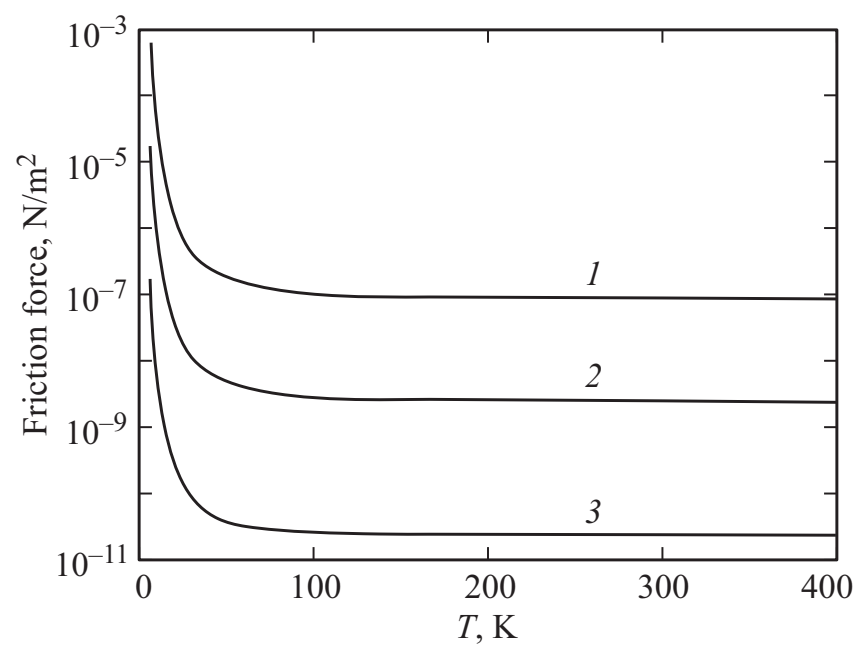

Рис. 4. Сила трения как функция температуры в приближении Друде (44) с учетом зависимости времени релаксации электронов $\tau$ от температуры $T(V=1 \mathrm{~m} / \mathrm{s})$. Кривые $1-3$ соответствуют ширине щели $a=10,100,1000 \mathrm{~nm}$.

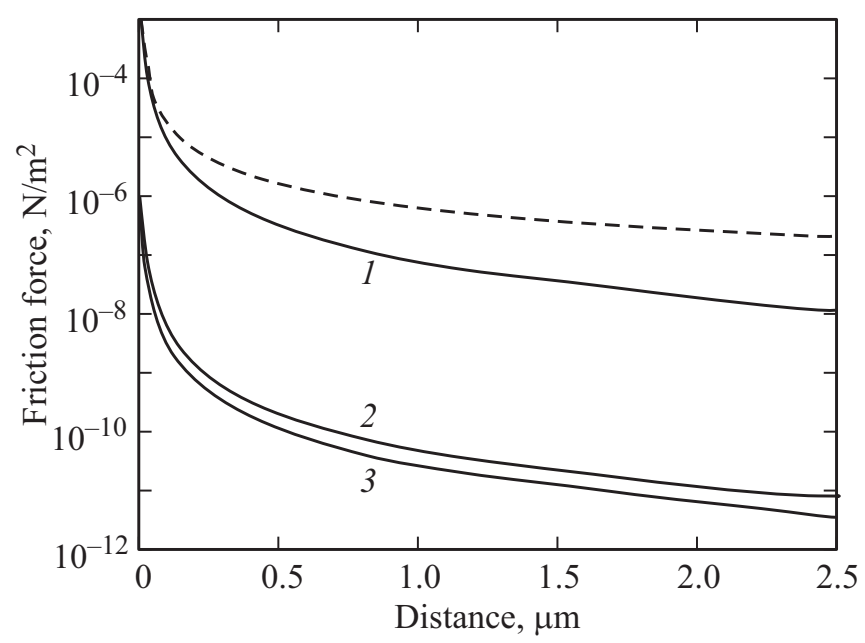

Рис. 5. Сила трения как функция расстояния в приближении Друде (44) с учетом зависимости времени релаксации электоронов $\tau$ от температуры $T(V=1 \mathrm{~m} / \mathrm{s})$. Кривые $1-3$ соответствуют температурам 5, 100 и $300 \mathrm{~K}$. Пунктирная кривая - аппроксимация $F_{x} \sim a^{-1.3}$.

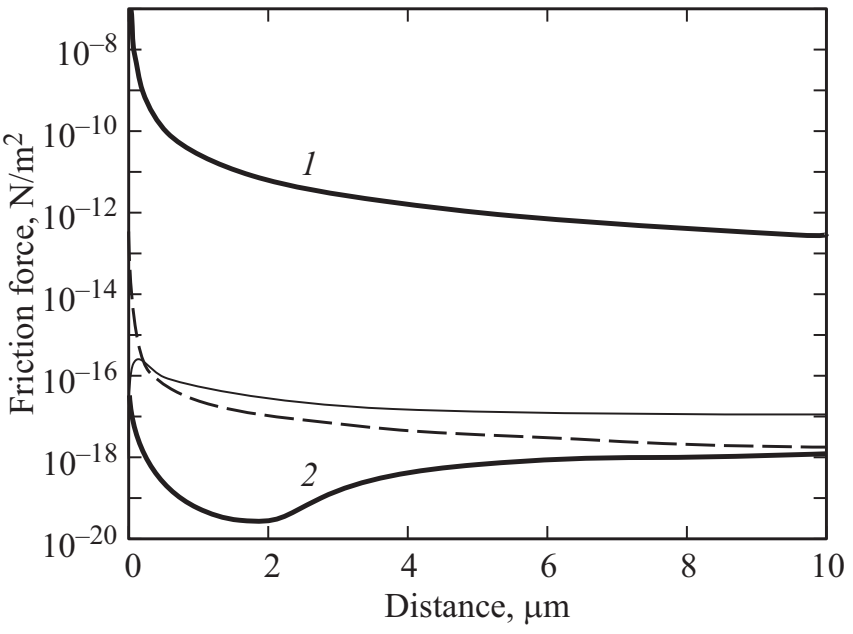

Рис. 6. Различные вклады в силу трения в приближении Друде (44). Сплошные линии 1 и $2-$ вклады магнитных ближнепольных и однородных волн, тонкие сплошная и пунктирная линии - вклады электрических ближнепольных и однородных волн $(T=300 \mathrm{~K}, V=1 \mathrm{~m} / \mathrm{s})$.

нородных и однородных волн - тонкими сплошной и пунктирной линиями. При других температурах соотношение различных вкладов качественно не изменяется. Поведение $F_{x}$ при температурах $T<1 \mathrm{~K}$ необходимо исследовать более детально в связи с различными механизмами и альтернативными теоретическими моделями для коэффициентов отражения металлов при низких температурах [9,21-24] (см. далее).

Представляет также интерес сравнить значения сил трения с экспериментальными измерениями диссипативных сил в эксперименте [25], соответствующем геометрии сферического зонда $(\mathrm{Au})$ с радиусом кривизны $1 \mu \mathrm{m}$, движущегося над плоской поверхностью слюды, покрытой пленкой золота: $\sim 1.5 \cdot 10^{-13} \mathrm{~N}$ при $a=10 \mathrm{~nm}, V=1 \mathrm{~m} / \mathrm{s}$. Используя локально-плоское приближение Дерягина [26], сила трения в вакуумном контакте сферического тела с пластиной оценивается как $F_{s p} \approx \pi R a F_{x}(a)$. Из наших расчетов следует $F_{x}(a)=1.6 \mathrm{~N} / \mathrm{m}^{2}$ и $F_{s p} \sim 0.5 \cdot 10^{-13} \mathrm{~N}$ при тех же условиях и $T=1 \mathrm{~K}$, причем зависимость силы $F_{s p}$ от расстояния (см. пунктирную кривую на рис. 5) близка к экспериментальной: $F_{s p} \sim a^{-\alpha}$ при $\alpha=1.3 \pm 0.3$. Однако в [25] измерения проводились при комнатной температуре, а при понижении температуры до $77 \mathrm{~K}$ величина диссипативной силы уменьшалась в шесть раз, т. е. температурная зависимость не согласуется с нашими расчетами в рамках флуктуационно-электромагнитного механизма. В свою очередь, вклад электростатического (из-за наличия пятен заряда на поверхностях) и флуктуационно-электромагнитного трения в этом случае, по оценкам $[10]$, оказывается на восемь порядков величины меньше экспериментальных значений при $T=300 \mathrm{~K}$, и еще более уменьшается при понижении температуры из-за возрастания проводимости. Таким образом, вопрос о природе диссипативных сил, измерявшихся в [25], пока остается открытым. 


\section{6. Обсуждение и выводы}

В п. 3 и 4 мы показали, что фундаментальные выражения для скорости радиационного теплообмена и силы трения двух толстых пластин, разделенных плоской щелью, полученные в работах Левина, Рытова и Полевого, полностью согласуются с результатами других авторов в случае нерелятивистского относительного движения пластин. При этом численный расчет силы трения для хороших металлов приводит к значениям в $10^{7}$ раз выше по сравнению с первоначальной оценкой Полевого (при комнатных температурах). Случай релятивистского движения представляет, главным образом, академический интерес и требует специального анализа. Рассмотрение сил трения для шероховатых поверхностей или поверхностей с наличием пленок не входило в нашу задачу, и, в принципе, может быть выполнено аналогично тому, как это делается при вычислении сил Казимира [21] или в расчетах диссипативных сил [10].

В свою очередь, из результатов расчетов п. 5 следует, что проведение экспериментов по измерению тангенциальной силы ван-дер-ваальсового трения становится вполне реальной задачей при использовании пробных сферических тел с радиусом 50-100 $\mu \mathrm{m}$, применявшихся при измерениях сил Казимира [22-24]. Причем сила трения возрастает на несколько порядков величины с увеличением проводимости металлов (при температурах ниже $50 \mathrm{~K}$ ), если время релаксации электронов в формуле Друде определяется законом Блоха-Грюнайзена. Между тем правомерность использования формулы Друде для описания диэлектрических свойств металлов при низких температурах подвергается критике в связи с ее недостатками при вычислении силы Казимира (см. [2224] и ссылки). В частности, констатируется, что корректное описание силы Казимира между металлическими пластинами достигается с использованием плазменной модели диэлектрической проницаемости, когда $\tau=\infty$ в формуле Друде (44). В этой связи заметим, что формальное применение плазменной модели при вычислении силы трения приводит к полному обнулению вклада ближнепольных мод в формулах (20), (23) или (42), т.е. наиболее значительного по величине вклада в трение. Тем не менее возрастание силы трения при низких температурах связано именно с увеличением величины $\tau$. С другой стороны, следует иметь в виду, что область частот, вносящих наибольший вклад в силу Казимира, существенно отличается (в большую сторону) от области частот, определяющих силу трения, для которой важны частоты $\omega \ll \omega_{w}$. Это связано с ролью планковского фактора $1 / x^{2}$ в (42) при $x \ll 1$. Благодаря этому, на наш взгляд, реальный ход силы трения при низких температурах является более сильным индикатором для проверки моделей диэлектрической проницаемости по сравнению с измерениями сил Казимира. В отличие от силы Казимира, для которой можно указать диапазон расстояний, на которых температурный вклад превалирует, сила трения при $T \neq 0$ целиком обусловлена виновскими частотами (т. е. имеет тепловой характер), а квантовая сила трения при $T=0$ имеет совсем другую зависимость от скорости [13,18-20] и численно на много порядков меньше (при тех же остальных параметрах). В этой связи отсутствие тепловых поправок в экспериментах по измерению сил Казимира [22-24] не дает оснований для аналогии с поведением силы трения. Для последней, как следует из рис. 4, влияние температуры в достаточно широком интервале $50<T<400 \mathrm{~K}$ мало и не зависит от расстояния $a$ между пластинами.

\section{Приложение}

При вычислении интегралов в (30), (34), (38) и (40) с функцией Друде (44) целесообразно записать $\varepsilon_{D}(\omega)$ в виде

$$
\begin{gathered}
\varepsilon_{D}(\omega=|\varepsilon| \exp (i \tilde{\phi}), \\
|\varepsilon|=\left[\left(\frac{1+\kappa_{w}^{2} x^{2}-\kappa_{p}^{2}}{1+\kappa_{w}^{2} x^{2}}\right)^{2}+\frac{\tilde{\lambda}^{2}}{x^{2}\left(1+\kappa_{w}^{2} x^{2}\right)^{2}}\right]^{1 / 2}, \\
\tilde{\phi}=\arctan \left(\frac{\kappa_{p}^{2}}{\kappa_{w} x\left(1+\kappa_{w}^{2} x^{2}-\kappa_{p}^{2}\right)}\right),
\end{gathered}
$$

где $\tilde{\lambda}=\kappa_{p}^{2} / \kappa_{w}$. Соответственно, формулы (31), (33), (35), (37)-(41) для величин $U_{1,2}$ и $\phi_{1,2}$ приводятся к виду

$$
\begin{aligned}
U_{1}= & {\left[\left(y^{2}+\frac{\lambda_{a}^{2} \kappa_{p}^{2} x^{2}}{1+\kappa_{w}^{2} x^{2}}\right)^{2}+\frac{\lambda^{2} x^{2}}{\left(1+\kappa_{w}^{2} x^{2}\right)^{2}}\right]^{1 / 4} } \\
\phi_{1}= & -0.5 \arctan \left[\frac{\lambda x}{y^{2}\left(1+\kappa_{w}^{2} x^{2}\right)+\lambda_{a}^{2} \kappa_{p}^{2} x^{2}}\right] \\
U_{2}= & {\left[\left(-y^{2}+\frac{\kappa_{p}^{2} x^{2}}{1+\kappa_{w}^{2} x^{2}}\right)^{2}+\frac{\tilde{\lambda}^{2} x^{2}}{\left(1+\kappa_{w}^{2} x^{2}\right)^{2}}\right]^{1 / 4}, } \\
\phi_{2}= & -0.5 \arctan \left[\frac{\tilde{\lambda} x}{\kappa_{p}^{2} x^{2}-y^{2}\left(1+\kappa_{w}^{2} x^{2}\right)}\right] \\
I_{\varepsilon 1}= & \int_{0}^{\infty} d y y^{3}\left(y^{2}+\lambda_{a}^{2} x^{2}\right) U_{1}^{2}|\varepsilon|^{-2}\left|Q_{\varepsilon 1}\right|^{-2} \sin ^{2}\left(\phi_{1}-\tilde{\phi}\right), \\
\left|Q_{\varepsilon 1}\right|^{2}= & \mid\left(y^{2}+U_{1}^{2}|\varepsilon|^{-2} \exp \left(2 i\left(\phi_{1}-\tilde{\phi}\right)\right)\right) \sinh (y) \\
& +\left.2 y U_{1}|\varepsilon|^{-1} \exp \left(i\left(\phi_{1}-\tilde{\phi}\right) \cosh (y)\right)\right|^{2}, \\
& \quad x \\
I_{\varepsilon 2}= & \int_{0} d y y^{3}\left(x^{2}-y^{2}\right) U_{2}\left|Q_{\varepsilon 2}\right|^{-2}|\varepsilon|^{-2} \sin { }^{2}\left(\phi_{2}-\tilde{\phi}\right), \\
= & \mid\left(y^{2}+U_{1}^{2}|\varepsilon|^{-2} \exp \left(2 i\left(\phi_{1}-\tilde{\phi}\right)\right)\right) \sin \left(\lambda_{a} y\right)
\end{aligned}
$$

где $\lambda=\kappa_{p}^{2} \lambda_{a}^{2} / \kappa_{w}$. Выражения (32), (34), (36) и (42) должны использоваться с учетом сделанных модификаций для $U_{1,2}$ и $\phi_{1,2}$. 


\section{Список литературы}

[1] М.Л. Левин, С.М. Рытов. Теория равновесных тепловых флуктуаций в электродинамике. Наука, М. (1967).

[2] С.М. Рытов. Теория электрических флуктуаций и теплового излучения. АН СССР, М. (1953).

[3] М.Л. Левин, В.Г. Полевой, С.М. Рытов. ЖЭТФ 79, 2087 (1980).

[4] В.Г. Полевой. Теплообмен флуктуационным электромагнитным полем. Наука, М. (1990).

[5] В.Г. Полевой. ЖЭТФ 98, 1990 (1990).

[6] D. Polder, M. Van Hove. Phys. Rev. B 4, 3303 (1971).

[7] J.J. Loomis, H.J. Maris. Phys. Rev. B 50, 18517 (1994).

[8] K. Park, Z. Zhang. Frontiers Heat Mass Transfer 4, 013001 (2013).

[9] V.B. Bezerra, G. Bimonte, G.L. Klimchitskaya, V.M. Mostepanenko, C. Romero. Eur. Phys. J. C 52, 701 (2007).

[10] A.I. Volokitin, B.N.J. Persson. Rev. Mod. Phys. 79, 1291(2007).

[11] B.N.J. Persson, Zhang Zhenyu. Phys. Rev. B 57, 7327 (1998).

[12] A.I. Volokitin, B.N.J. Persson. J. Phys. C 11, 345 (1999).

[13] J.B. Pendry. J. Phys. C 9, 10301 (1997).

[14] V.E. Teodorovich. Proc. Roy. Soc. London A 362, 71 (1978).

[15] T.G. Philbin, U. Leonhardt. New J. Phys. 11, 03035 (2009); arXiv: 094.2148.

[16] A.I. Volokitin, B.N.J. Persson. New J. Phys. 11, 033035 (2009).

[17] J.B. Pendry. New J. Phys. 12, 033028 (2010).

[18] J.S. Høye, I. Brevik. Entropy 15, 3045 (2013).

[19] K.A. Milton, J.S. Hoye, I. Brevik. Symmetry 8, 29 (2016).

[20] G.V. Dedkov, A.A. Kyasov. Phys. Usp. 187, 559 (2017).

[21] K.A. Milton, R. Guerodt, G.L. Ingold, A. Lambrecht, S. Reynaud. J. Phys. Condens. Matter 27, 214003 (2015).

[22] M. Bordag, G.L. Klimchitskaya, U. Mohideen, V.M. Mostepanenko. Advances in the Casimir effect. Oxford Univ. Press, Oxford, UK (2009).

[23] C.-C. Chang, A.A. Banishev, R. Castillo-Garza, G.L. Klimchitskaya, V.M. Mostepanenko, U. Mohideen. Phys. Rev. B 85, 165443 (2012).

[24] A.A. Banishev, G.L. Klimchitskaya, V.M. Mostepanenko, U. Mohideen. Phys. Rev. B 88, 155410 (2013).

[25] B.C. Stipe, H.J. Mamin, T.D. Stowe, Y.W. Kenny, D. Rugar. Phys. Lett. 87, 9, 096801 (2001).

[26] B.V. Derjaguin. Kolloid Z. 69, 155 (1934).

Редактор Т.Н. Василевская 